\title{
Foliations Transverse to Triangulations of 3-Manifolds
}

\author{
DANNY CALEGARI
}

\begin{abstract}
We investigate the combinatorial analogues, in the context of normal surfaces, of taut and transversely measured (codimension 1) foliations of 3-manifolds. We establish that the existence of certain combinatorial structures, a priori weaker than the existence of the corresponding foliation, is sufficient to guarantee that the manifold in question satisfies certain properties, e.g. irreducibility. The finiteness of our combinatorial structures allows us to make our results quantitative in nature and has (coarse) geometrical consequences for the manifold. Furthermore, our techniques give a straightforward combinatorial proof of Novikov's theorem.
\end{abstract}

\section{Introduction.}

In this paper we study some of the relationships between normal surfaces and foliations. Our general approach is to discuss what combinatorial structure on a triangulation of a 3-manifold is sufficient to guarantee the existence of a foliation in "normal form" with respect to the triangulation. i.e. we want every leaf of the foliation to be a normal surface away from the vertices of $M$. Or, if we cannot guarantee such a foliation, we consider what combinatorial structure is sufficient to guarantee properties of $M$ that would be a consequence of the existence of a foliation.

The combinatorial structure we describe consists of a choice of orientation for each edge of the 1-skeleton $M^{1}$. That there exists a transverse foliation locally amounts to a local condition on the star of each vertex. The problem of the global existence of a foliation seems to be a very hard problem, and we are not able to treat it effectively except in some special cases. In particular, the related problem of when a branched surface carries (abstractly) a lamination was shown to be algorithmically unsolvable, by Lee Mosher (see [Ga]), although it is not known whether the problem is still unsolvable if the branched surface is given together with an embedding in a 3-manifold. 
In the first section, we are able to give precise conditions for the existence of a transversely measured normal foliation. Our condition says that directed loops in the 1-skeleton (with respect to our orientation) must lie in an open half-space of homology. As a corollary, we are able to give an elementary combinatorial proof that a 3-manifold admitting a taut foliation in which the transverse loops lie in an open half-space of homology, is a surface bundle over $S^{1}$.

In the second section we treat the converse question, of when a foliation of $M$ by closed surfaces can be put in normal form with respect to a triangulation. We show that if one can make the 1-skeleton transverse to the foliation and have at least one incoming and one outgoing edge from each vertex, then the foliation can be isotoped rel. its intersection with the 1 -skeleton to be in normal form.

In the next two sections we discuss when a triangulation admits a taut foliation in normal form. If a choice of orientations on the 1-skeleton as above admits a transverse foliation locally, if every oriented loop is homotopically essential, and if in addition the 1-skeleton is recurrent, thought of as a directed graph, then we are able to show that the universal cover of $M$ can be given a foliation in normal form such that each leaf is incompressible. As a corollary, we see that a manifold admitting such a combinatorial structure is either irreducible or $S^{2} \times S^{1}$. This is a generalization of Novikov's theorem, and it remains to be seen whether the combinatorial structure so described is more general than that of the existence of a taut foliation. Our methods give new information even about 3-manifolds admitting taut foliations, showing that any deformation of the foliation in the universal cover which is "roughly equivariant" (i.e. preserves the normal disk types in each tetrahedron) also has incompressible leaves. Moreover, our technique gives an elementary (combinatorial) proof that circles transverse to leaves of a taut foliation are homotopically essential.

In fact, we can make our results quantitative, and show that the leaves in the universal cover satisfy the same kind of isoperimetric inequalities that minimal surfaces in $M$ satisfy. Our argument here uses the finiteness of the combinatorial structure, which makes uniform geometric estimates almost effortless.

In the penultimate section, we weaken our hypotheses considerably and show that we can still get a strong result. In particular, we weaken the condition that oriented loops be homotopically essential to the condition that oriented loops of length $\leq k_{1}$ bound no simplicial disks of simplicial area $\leq k_{2}$, for constants $k_{1}, k_{2}$ depending on the triangulation and the choice of 
orientation. With this hypothesis, we can nevertheless show that $\pi_{2}(M)=1$ or $M=S^{2} \times S^{1}$.

This result is interesting insofar that it shows that even the "local" existence of a foliation transverse to the 1-skeleton is enough to get topological information about the manifold.

In particular, we expect that this condition is much more general than the existence of a foliation in normal form on $M$ compatible with the orientations. This brings to mind a question of D. Gabai in [Ga], namely: "Do there exist useful branched surfaces which do not carry anything"? Though we do not demonstrate the non-existence of foliations compatible with our combinatorial structures, nevertheless, we do not need to produce a globally compatible foliation to find the combinatorial structure "useful".

We adhere to the convention, it what follows, even if we do not mention it explicitly, that every foliation is oriented, co-oriented, and smooth. For simplicity, and to avoid headaches, we have not investigated the extent to which these conditions can be dropped.

I would like to thank Andrew Casson for his patience, his comments, and his suggestions regarding the following material. I am also grateful to the referee for some excellent observations and comments.

\section{Triangulations and maps to $S^{1}$.}

Let $M$ be a compact 3-manifold, and let $\mathcal{T}$ be a triangulation of $M$. A positive orientation on $\mathcal{T}$ is a choice of orientation on each edge of $\mathcal{T}^{1}$, and a choice of $\alpha \in H^{1}(M ; \mathbb{R})$ such that

- For each loop $\gamma \subset \mathcal{T}^{1}$ with $\gamma$ positively oriented, $\alpha([\gamma])>0$, where $[\gamma]$ denotes the image of $\gamma$ in $H_{1}(M ; \mathbb{R})$

- For every vertex $v \in \mathcal{T}^{1}$ let $o(v)$ be the set of vertices $w$ such that there is an oriented edge from $v$ to $w$, and let $i(v)$ be the set of vertices $w$ such that there is an oriented edge from $w$ to $v$. Then there is a connected subgraph of $\operatorname{link}(v)$ whose vertices are exactly $o(v)$, and a connected subgraph of $\operatorname{link}(v)$ whose vertices are exactly $i(v)$.

We call a choice of orientation for each edge of a cell complex $C$ a direction on $C$. Maps between directed $C W$ complexes are orientation-preserving if they preserve the orientation on each edge.

Lemma 2.1. Let $C$ be a complex together with a direction. There is an orientation-preserving immersion $f: C^{1} \rightarrow S^{1}$ which is affine on each edge 
(with respect to the standard affine structure on $S^{1}$ ), and extends to all of $C$ iff all oriented cycles in $C^{1}$, considered as elements of $H_{1}(C)$, are contained in an open half-space.

Proof. An orientation-preserving immersion $f: C^{1} \rightarrow S^{1}$ is determined by a collection of real values $x_{i}>0$, one for each edge $e_{i}$, such that we have

$$
\sum_{e_{i} \in \gamma} x_{i} \in \mathbb{Z}
$$

for each oriented cycle $\gamma$. This map extends to $C^{2}$ iff for each disk $D \in C^{2}$, the boundary $\partial D$ is mapped to $S^{1}$ null-homotopically. That is, writing the boundary $\partial D$ as a union of positively and negatively oriented edges,

$$
E_{j}=\sum_{e_{i} \in \partial D_{j}} x_{i}-\sum_{-e_{i} \in \partial D_{j}} x_{i}=0
$$

where our notation is meant to indicate whether the orientation of $e_{i}$ agrees or disagrees with the orientation of $\partial D$ induced by some arbitrary orientation on $D$.

It is a fact that the collection of equalities $E_{j}=0$ has a solution with all $x_{i}$ positive iff there is no linear combination of $\sum_{j} v_{j} E_{j}$ such that

$$
\sum_{j} v_{j} E_{j}=\sum_{i} c_{i} x_{i}
$$

with all $c_{i}$ non-negative, and at least one $c_{i}$ positive. For, let $H$ be the subspace of $\mathbb{R}^{n}$ spanned by the vectors $E_{i}$. Then the orthogonal subspace $H^{\perp}$ is precisely the set of solutions to the equations $E_{i}=0$. If $K$ denotes the convex cone where all $x_{i}>0$, then vectors $v \in K \cup-K$ are characterised by the property that $v^{\perp}$ does not intersect $K$. From certain perspectives, this is just the finite dimensional version of the Hahn-Banach theorem.

Notice that, thought of as an element of the set of 1-chains on $M$, each. $E_{j}$ is a boundary. Therefore $\sum_{j} v_{j} E_{j}=\partial a$ for some 2-chain $a$. But any sum. $\sum_{i} c_{i} x_{i}$ with all $c_{i}$ non-negative that represents a 1-cycle must consist of a non-negative sum of positively oriented cycles $\gamma \in C^{1}$. Since such cycles all lie in the same open half-space of $H_{1}$, a positive combination of them cannot be a boundary.

Having found a positive solution, we may find one with rational coefficients, and therefore by scaling, with integral coefficients.

Finally, since $S^{1}$ is aspherical, any map on $C^{2}$ extends to $C$. 
With this lemma we may establish

Theorem 2.2. If $M$ admits a triangulation with a positive orientation, then $M$ is a surface bundle over $S^{1}$ and there is a projection map $M \rightarrow S^{1}$ which is affine on each simplex.

Proof. Let $\mathcal{M}$ denote a geometric model for $M$ constructed by letting each tetrahedron of the triangulation be a regular Euclidean tetrahedron with side lengths equal to 1 .

By our lemma, the first condition implies that there is an orientationpreserving immersion $f: \Gamma \rightarrow S^{1}$ which extends to all of $\mathcal{T}$. By a genericity assumption, we insist that the images of all the vertices are sent to distinct points of $S^{1}$. The restriction of $f$ to the 1-skeleton of each tetrahedron $\Delta$ therefore lifts to a map $\tilde{f}: \Delta^{1} \rightarrow \mathbb{R}$. We extend $\tilde{f}$ to the entire tetrahedron $\Delta$ by requiring it to be affine. It is clear that this can be done compatibly to give an piecewise-affine map $\mathcal{M} \rightarrow S^{1}$. We claim that this map is the projection map from the total space of a surface bundle to the base space. To see this, it suffices to show that the foliation of $\mathcal{M}$ by the preimages of points in $S^{1}$ is non-singular.

Notice that there is only one possible orientation for each $\Delta^{1}$, up to isomorphism, and it is clear that this orientation induces a non-singular foliation on $\Delta$. This foliation pieces together compatibly along faces and edges. It remains to check that it is non-singular at vertices.

Let $v$ be a vertex of the triangulation, and $\operatorname{star}(v)$ denote the star of $v$. Then $f$ lifts to $\tilde{f}: \operatorname{star}(v) \rightarrow \mathbb{R}$, since topologically, $\operatorname{star}(v)$ is a $B^{3}$ which is simply-connected. $\operatorname{link}(v)$ is a triangulated $S^{2}$. Then $\left.f\right|_{\operatorname{link}(v)}$ is non-degenerate away from the vertices, and its level sets away from these consist of a disjoint union of circles.

If $\lambda$ is the leaf containing $v$, then $\lambda \cap \operatorname{star}(v)$ is the cone on the set of $p \in \operatorname{link}(v)$ where $\tilde{f}(p)=\tilde{f}(v)$. We must show that this is a circle. By genericity, this set is a disjoint union of circles. Let $S$ be a circle in $\operatorname{link}(v)$ separating a maximal graph whose vertices are $o(v)$ from a maximal graph whose vertices are $i(v)$. Call these two graphs $\Gamma_{o}$ and $\Gamma_{i}$. Such a circle exists, since the two graphs are disjoint, and any two connected closed subsets of $S^{2}$ are separated by an embedded circle.

The union of simplices in $\operatorname{link}(v)$ intersecting $S$ non-trivially is an open annulus whose boundary is contained in $\Gamma_{i} \cup \Gamma_{o}$, and it is clear that we can write the annulus as $S^{1} \times I$ where $\tilde{f}$ is monotonically increasing on each $p \times I$. Since the value of $\tilde{f}$ on one boundary component of this annulus is 
strictly greater than $\tilde{f}(v)$, and strictly less than it on the other component, $\tilde{f}^{-1}(\tilde{f}(v)) \cap S^{1} \times I$ is a single circle. Now for any $p$ in the complement of this annulus, $\tilde{f}(p)$ is a convex combination of values strictly larger than, or a convex combination of values strictly smaller than $\tilde{f}(v)$ - namely the values of $\tilde{f}$ on the vertices on the appropriate side. Hence $\tilde{f}^{-1}(\tilde{f}(v)) \cap \operatorname{link}(v)$ is a single circle, and the foliation is nonsingular at $v$.

Corollary 2.3. Let $M$ be a 3-manifold with a taut oriented, co-oriented, smooth foliation $\mathcal{F}$ and let $\alpha \in H^{1}(M ; \mathbb{R})$ be such that for every transverse, positively oriented cycle $\gamma$ the inequality $\alpha([\gamma])>0$ is true. Then $M$ is a surface bundle over $S^{1}$.

Proof. To see that $M$ fibers over $S^{1}$, just take a very fine triangulation of $M$ with all edges transverse to $\mathcal{F}$. Orient the edges according to the coorientation on $\mathcal{F}$, and make the edges "straight" enough that the induced orientation on the 1-skeleton of the triangulation is a positive triangulation.

Essentially, if we restrict to a small $I^{3}$ foliated by $z=$ const., we want the triangulation in that small $I^{3}$ to be by approximately affine tetrahedra with respect to the affine structure on $I^{3}$. The condition is automatically satisfied for affine triangulations, with edges oriented by a co-orientation on an affine foliation.

For, let $p, q$ be two vertices in $o(v)$. Let $\pi$ be the plane spanned by $p, q, v$. This plane intersects $\operatorname{link}(v)$ in a circle, $\operatorname{since} \operatorname{star}(v)$ is star-shaped with center $v$. Let $\gamma$ be the arc of this circle joining $p$ to $q$ such that the value of $z$ on $\gamma$ is greater than the value of $z(v)$. Then either $\gamma$ is an edge of the 1-skeleton, in which case $\Gamma_{o}$ connects $p$ to $q$, or it intersects the 1-skeleton at some first point $m$ in the interior of an edge $e$ If the latter case, $z(m)>z(v)$, so there is a vertex $r$ on $e$ with $z(r)>z(m)>z(v)$. We "slide" $\gamma$ along $m$ to $r$, and continue inductively to produce a path in $\Gamma_{o}$ connecting $p$ to $q$, so $\Gamma_{o}$ is connected. Similarly, $\Gamma_{i}$ is connected.

Since Riemannian manifolds are locally almost affine, this can be done compatibly over the entire triangulation. More precisely, since $\mathcal{F}$ is smooth, we can cover $M$ with co-ordinate patches such that in each co-ordinate patch, $\mathcal{F}$ is a foliation of $\mathbb{R}^{3}$ by level sets of the form $z=$ const. and the co-ordinate transformations are "almost" linear. Here "almost" means sufficiently close that the triangulation can be straightened to an affine triangulation in each patch without disturbing the local combinatorial structure. Essentially, we just need to pick a triangulation by sufficiently "squat" simplices. A rigorous proof of this fact can be found in $[\mathrm{Be}]$, where it is attributed originally to 
Thurston.

Since a smooth foliation locally resembles such an affine foliation to first order, this can be done compatibly over the entire manifold.

The triangulation of $M$ is therefore positively oriented, and $M$ is a surface bundle over $S^{1}$, as required.

Remark 2.1. In fact, $\mathcal{F}$ as above carries a transverse measure $\mu$ such that $\mu(\gamma)=\alpha([\gamma])$ for any transverse, positively oriented cycle $\gamma$. Suppose, for example, that some leaf $\lambda \in \mathcal{F}$ is dense in $M$. Then for a given transversal $t$, there are points in $t \cap \lambda$ arbitrarily near the endpoints of $t$. These can be joined up by a path in $\lambda$ to give a cycle. Evaluating $\alpha$ on this cycle, and taking the supremum over all pairs of points which tend toward the ends of $t$, we get $\mu(t)$. This is positive, since it is greater than the value of $\alpha$ on some positive transverse cycle, which is $>0$. A choice of a different path in $\lambda$ might give a different cohomology class, but these would differ by a cohomology class carried by a loop in $\lambda$, and $\alpha$ evaluated on this class is necessarily 0 . If we homotope $t$, keeping its endpoints on the same leaf, we can join it up by a path in $\lambda$ homotopic to the original path, and therefore giving the same value when $\alpha$ is evaluated on it. Finally, if we write $t$ as a union of two intervals, then a cycle joining up $t$ is homologous to a sum of two cycles, each joining up one of the sub-intervals of $t$.

If we pick some fine triangulation, and an associated map to $S^{1}$, the pullback of the angular measure on $S^{1}$ to $M$ is "approximately" a transverse measure for $\mathcal{F}$. It is positive on any monotone path in the 1 -skeleton of the triangulation. However, monotone paths which are "nearly horizontal" are not generally approximated by a monotone path in the 1-skeleton. By including more and more monotone paths in the 1-skeleton, we can approximate $\mu$ more and more closely. In fact, the more elements of $H_{1}$ that are carried by monotone cycles in the triangulation, the less flexibility we have in choosing the homotopy class of our map to $S^{1}$, and the better the pullback measure approximates $\mu$.

Remark 2.2. Notice that there is nothing inherently 3-dimensional about our theorem. If $M$ is an arbitrary manifold, and $\mathcal{F}$ a smooth codimension one co-oriented foliation such that every transverse cycle lies in a half-space of $H_{1}$, then if we choose a very fine triangulation transverse to the foliation which can be straightened to an affine triangulation in every co-ordinate chart without disturbing the combinatorial structure, and we orient the 1skeleton according to $\mathcal{F}$, then the piecewise affine map to $S^{1}$ guaranteed by the lemma induces a non-singular foliation everywhere, and exhibits the 
manifold as a bundle over $S^{1}$. We prove our theorem in the 3-dimensional case only because that is our interest for applications.

Remark 2.3. In [Su], D. Sullivan proves the following conjecture of R. Edwards: if $M$ is a foliated manifold with all leaves compact, such that the homology classes represented by the leaves lie in an open half-space of homology, for the appropriate dimension, then $M$ is transversely measured. Notice that if the foliation is of codimension 1, then this condition implies the condition of the corollary above. Our proof owes something to Sullivan's approach - in particular, the key result from linear algebra that we use is a finite dimensional version of the Hahn-Banach theorem, which in its general form is essential in setting up the machinery for Sullivan's theorem.

\section{Normal Form for Surface Bundles.}

The results of the last section suggest the question of when a triangulation of a surface bundle admits a positive orientation.

Theorem 3.1. Let $M$ be a surface bundle over $S^{1}$ with projection map $r: M \rightarrow S^{1}$. Let $\mathcal{T}$ be any triangulation of $M$ such that the star of each vertex is an embedded $B^{3}$ in $M$, and such that the following two conditions are satisfied:

- each edge is monotone with respect to $r$ and oriented according to the orientation in the circle direction

- there is an outgoing edge and an incoming edge for every vertex

Assume that the genus of the surface is at least 1.

Then the induced orientation of the edges is a positive orientation.

Proof. We lift this triangulation to a triangulation of the universal cover, which is $\mathbb{R}^{3}$ foliated by planes. Notice that the lift of the triangulation also satisfies these two properties. By abuse of notation, we denote by $r$ the map $r: \tilde{M} \rightarrow \mathbb{R}$ whose preimages are the lifts of surfaces.

By the results of the first section, there is an (equivariant) map $f: \tilde{M} \rightarrow$ $\mathbb{R}$ affine on each simplex, and agreeing with $r$ on the 1-skeleton.

We can further assume a non-degeneracy condition, namely that for each pair of vertices $v, w, f(v) \neq f(w)$.

Then

$$
C_{1} \cup C_{2} \cup \cdots \cup C_{i}=f^{-1}(f(v)) \cap \operatorname{link}(v)
$$


is a non-empty disjoint collection of circles. Suppose there are at least two circles, $C_{1}, C_{2}$.

The union of the simplices intersecting $C_{i}$ is an open annulus, and we label its boundary components $u_{i}$ and $l_{i}$, where $f\left(l_{i}\right)<f\left(C_{i}\right)<f\left(u_{i}\right)$ for each $i$. It is possible that $u_{1} \cap u_{2}$ or $l_{1} \cap l_{2}$ are non-empty, but they cannot both be non-empty, for otherwise $C_{1}$ would be a non-separating circle in $\operatorname{link}(v)$, an absurdity. WLOG, say that $u_{1} \cap u_{2}$ is empty. Let $U_{i}$ be the disk consisting of the region of $\operatorname{link}(v)$ bounded by $l_{i}$ and containing $C_{i}$. We assume $U_{1}$ and $U_{2}$ are disjoint, for otherwise there is an annulus between $C_{1}$ and $C_{2}$ and a circle $C_{i}$ a meridian of this annulus, and we can replace one of $C_{1}, C_{2}$ by $C_{i}$ if necessary so that this is satisfied.

Let $x_{i}$ be the vertex attaining the maximum value of $f$ on $U_{i}$. Since $l_{i}$ is an unknotted circle, and since $f\left(l_{i}\right)=r\left(l_{i}\right)<r(v)=f(v)$ on this circle, there is an embedded disk $D_{i}$ with $r\left(D_{i}\right)<r(v)$ whose boundary is $l_{i}$. Since $D_{i}$ and $U_{i}$ are embedded with a common boundary, we can arrange that their intersection is a collection of circles. We perform disk exchanges on these circles to produce an embedded surface $S_{i}$ made from pieces of $U_{i}$ and $D_{i}$, and containing $\operatorname{nbhd}\left(x_{i}\right) \cap U_{i}$. (In fact, we do not need to do these exchanges - we can simply take as $S_{i}$ the boundary of some complementary region containing $x_{i}$.)

By assumption, there is an infinite increasing ray contained in the 1skeleton emanating from $x_{i}$. Call this ray $\alpha_{i}$. Since the value of $f$ on $\alpha_{i}$ is greater than $f\left(x_{i}\right)$, this ray cannot intersect $S_{i}$ except at $x_{i}$. For, if it does so, it intersects $S_{i}$ in the 1-skeleton. But for every point on $S_{i}$, either $r$ or $f$ is less than $f\left(x_{i}\right)$, and therefore for the intersection of $S_{i}$ with the 1-skeleton, $f$ is less than $f\left(x_{i}\right)$. Hence $\alpha$ is entirely contained in some complementary region of $S_{i}$, and since it is infinite, this region is unbounded.

Moreover, there is an increasing edge $e_{i}$ from $v$ to $x_{i}$. This edge intersects $S_{i}$ only at $x_{i}$. For, it cannot intersect any piece of $D_{i}$, since the value of $r$ is less than $f(v)$ there. Also, it cannot intersect $U_{i}$ except at $x_{i}$, since $e$ intersects $\operatorname{link}(v)$ only at $x_{i}$. Therefore the union $\beta_{i}=e_{i} \cup \alpha_{i}$ is an infinite increasing ray which intersects $S_{i}$ exactly once. Hence $v$ is in the bounded complementary region of $S_{i}$.

Notice also that $e_{1}$ does not intersect $S_{2}$ at all, nor does $e_{2}$ intersect $S_{1}$, since again it can only intersect it in pieces of $D_{i}$, and there the value of $r$ is less than $f(v)=r(v)$. Therefore $x_{1}$ is contained in the bounded complementary region of $S_{2}$, and $x_{2}$ in the bounded complementary region of $S_{1}$. WLOG, $f\left(x_{1}\right)>f\left(x_{2}\right)$. But then $\alpha_{1}$ cannot intersect $S_{2}$, since on the intersection of $S_{2}$ with the 1-skeleton, $x_{2}$ attains the highest value of $f$. Hence $\alpha_{1}$ is bounded by $S_{2}$, which is a contradiction. 
Therefore there is exactly one circle $C_{1}$, and the leaf $f^{-1}(f(v)) \cap \operatorname{star}(v)$ is a non-singular disk, and the orientation on the edges is a positive orientation, as required.

Remark 3.1. We may think of this theorem as giving a kind of "normal form" for surface bundles with respect to a triangulation. In particular, if the bundle can be made "normal" with respect to the 1-skeleton, this theorem guarantees it can be made "normal" with respect to the entire triangulation.

Remark 3.2. The issue is to decide for what triangulations $\mathcal{T}$ of a surface bundle $M$ the 1-skeleton can be made transverse to the foliation by surfaces in such a way that each vertex has an outgoing and an incoming edge, with respect to some co-orientation on the foliation.

Let $r: M \rightarrow S^{1}$ be any map generic with respect to the 1 -skeleton of $\mathcal{T}$. Then there is a subdivision of the 1-skeleton to a finite graph $\Gamma$ such that each edge of $\Gamma$ is transverse to foliation (i.e. take as additional vertices of $\Gamma$ the critical points of $\left.r\right|_{\mathcal{T}^{1}}$ ). There are two issues to be resolved. The first is whether $r: \Gamma \rightarrow S^{1}$ is homotopic to a map monotone on each edge, and with an outgoing and an incoming edge from each vertex. The second is the issue of whether such a homotopy of $r$ can be realized by an isotopy of $\Gamma$ in $M$.

Such a homotopy can be decomposed into a collection of "local" moves, which consist of exchanging the order of neighboring vertices and re-orienting; any edge between the two of them. Let $v_{1}, v_{2}$ be the vertices in question, and suppose $r\left(v_{1}\right)<r\left(v_{2}\right)$. Let $\alpha$ be the segment of $S^{1}$ between $r\left(v_{1}\right)$ and $r\left(v_{2}\right)$ containing the image of no other vertex. Then $r^{-1}(\alpha)$ is homeomorphic to surface $\times I$, and $\Gamma \cap r^{-1}(\alpha)$ consists of a collection of monotone arcs from one boundary component to the other, together with the set of outgoing arcs rooted at $v_{1}$, and the set of incoming arcs rooted at $v_{2}$, which are points on opposite boundaries. It is clear that the only obstruction to performing an isotopy exchanging the order of $v_{1}$ and $v_{2}$ is whether or not the outgoing edges from $v_{1}$ "link" the incoming edges to $v_{2}$.

\section{Partial Orderings.}

Let $M$ be a closed 3-manifold, and let $\mathcal{T}$ be a triangulation of $M$. A direction on $M$ is a choice of orientation for each edge in the 1-skeleton $\mathcal{T}^{1}$ of the triangulation. A direction is a local orientation if it satisfies the conditions

1. for each vertex $v$ the maximal subgraphs $o(v)$ and $i(v)$ of $\operatorname{link}(v)$ whose vertices are, respectively, the outgoing and the incoming vertices from 
and to $v$, are nonempty and connected

2. the direction restricts to a total ordering on the vertices of each tetrahedron

3. the 1-skeleton is recurrent as a directed graph. That is, there is an increasing path from each vertex to each other vertex.

Example. On $S^{3}$, consider the Hopf vector field. This is a volume preserving flow, so any cone field which supports this vector field is recurrent. (for the definition of cone fields, see [Su]) If we take some sufficiently fine triangulation supported by such a cone field, the local orientation conditions will be satisfied, since locally there is a product structure given by the flow, which is transverse to our triangulation. Again, if the triangulation is sufficiently fine, it can be made recurrent, since the cone field is recurrent. However, there is no foliation transverse to this local orientation, for such a foliation would be taut by recurrence, which is impossible on $S^{3}$.

Since the vertices of each triangle in $\mathcal{T}^{2}$ are totally ordered, we can speak unambiguously of the long edge of any triangle, and also of the upper and lower short edges. We construct a directed graph $\Gamma$ associated to the direction whose vertices are edges of $\mathcal{T}^{1}$ and whose directed edges are the ordered pairs $\left(e_{i}, e_{j}\right)$ where $e_{j}$ is the long edge, and $e_{i}$ the upper or lower short edge of some triangle in $\mathcal{T}^{2} . \Gamma$ is expanding if it contains a pair of directed loops, one containing the edge $\left(e_{i}, e_{k}\right)$, one containing the edge $\left(e_{j}, e_{k}\right)$ where $e_{i}, e_{j}$ are the upper and lower short, and $e_{k}$ the long edge of some triangle.

A choice of orientation on the edges of a triangulation, or more generally a choice of orientation for the edges of a graph, determines a partial ordering on the vertices by declaring that $x \leq y$ iff there is an oriented path in the graph or 1-skeleton from $x$ to $y$. The partial orderings for the 1-skeleta of our compact manifolds $M$ will generally not be very interesting: recurrence implies that for any two elements $x, y$ both $x \leq y$ and $y \leq x$. However, if we pull back these orientations to the universal cover of $M$, the induced partial orderings are more interesting. We pursue this more vigorously in the next section.

Lemma 4.1. If $M$ admits a local orientation in which every oriented loop is homotopically essential, and if $\Gamma$ is expanding, then $\pi_{1}(M)$ has exponential growth. 
Proof. By the hypothesis, $\tilde{M}$ has no oriented loops in its 1-skeleton. But then the associated graph $\tilde{\Gamma}$ has no directed loops. Hence there is an infinite dyadic tree (the lift of the directed loops guaranteed by the condition that $\Gamma$ is expanding) which embeds in $\tilde{\Gamma}$. Hence $\tilde{M}$, and therefore $\pi_{1}(M)$, has exponential growth by the usual reason that the Cayley graph of $\pi_{1}(M)$ has the quasi-isometry type of $M$. (See for instance [Gr]).

The reason to introduce these definitions is given by the following lemma:

Lemma 4.2. A co-oriented tautly foliated 3-manifold admits a triangulation with a local orientation in which every oriented loop in the 1-skeleton is homotopically essential. Conversely, a foliation in normal form relative to a local orientation is taut.

Proof. As before, choose a triangulation such that $\mathcal{F}$ is in normal form with respect to the triangulation. Orient the edges of the triangulation according to the co-orientation on $\mathcal{F}$. This triangulation can be refined repeatedly until $M^{1}$ is recurrent.

The second statement is immediate.

Theorem 4.3. If $M$ admits a local orientation, then the induced orientation on any connected finite cover of $M$ is a local orientation.

Proof. The only non-trivial condition to check is recurrence. Suppose there exists a monotone path from $p$ to $q$ in the cover. Then this projects to a monotone path in $M$ which can be completed to a monotone loop, by recurrence. Then some power of this loop lifts to the cover, so there is a monotone path from $q$ to $p$. Hence $M^{1}$ breaks up into recurrent components. Since it is connected, there is only one such component.

Theorem 4.4. If $M$ admits a local orientation such that every oriented loop is homotopically essential, and $M$ has a finite cover with fundamental group $\mathbb{Z}$, then $M=S^{2} \times S^{1}$.

Proof. The finite cover, call it $N$, also has the property that every oriented loop is homotopically essential, and therefore equal to some power of the generator of $H_{1}(N)$. Suppose there are two oriented loops which represent $\alpha^{n}$ and $\alpha^{-m}$ for some positive integers $n, m$. Then there is another oriented 
loop representing $\alpha^{r}$ which connects two points on these loops. By composing loops, we can find an oriented loop representing $\alpha^{a n-b n+c r}$ for any positive integers $a, b, c$. But this implies that we can find an oriented loop representing the trivial element, a contradiction. Hence all oriented loops lie in an open half-space of $H_{1}(N)$, and by our theorem, $N$ is a surface bundle over $S^{1}$. Since $\pi_{1}(N)=\mathbb{Z}, N=S^{2} \times S^{1}$. Project a normal $S^{2}$ down to $M$. Then the edge weights determined by the (possibly immersed) image represent an embedded (possibly disconnected) co-oriented normal surface in $M$. If $M=\mathbb{R} P^{3} \# \mathbb{R} P^{3}$ then either component is separating and therefore violates the recurrence of $M$. Hence $M=S^{2} \times S^{1}$, as required.

\section{A Generalization of Novikov's Theorem.}

If $M$ admits a local orientation in which each directed loop is essential, then $\tilde{M}$ has no oriented loops. The results of our first section show that any compact subset $K \subset \tilde{M}$ admits a transverse measured foliation in normal form.

We now show that we can foliate $\tilde{M}$ globally.

Theorem 5.1. If $M$ admits a (not necessarily recurrent) local orientation in which each directed loop is essential, then $\tilde{M}$ admits a transverse measured foliation in normal form.

Proof. The idea is to collapse the partial ordering on the vertices of the 1-skeleton of $\tilde{M}$ to a total ordering, with some kind of geometric control, in order to construct a map $\tilde{M} \rightarrow \mathbb{R}$ which is an orientation-preserving embedding on each edge.

The proof is by induction. At stage $i$ we will have an infinite $C W 2$ complex $K_{i}$ where $K_{i}$ is obtained from $K_{i-1}$ by collapsing an interval, and where $K_{0}=\tilde{M}^{2}$, the 2-skeleton of the universal cover. Each 2-cell $D$ of $K_{i}$ will have the property that the attaching map $\partial D \rightarrow K_{i}$ is an embedding away from possibly finitely many points, and the induced orientation on $\partial D$ will have exactly one maximum and one minimum. Call the two oriented subarcs of $D$ the sides. We can arbitrarily call one the left side, and one the right side.

Let $v_{l}$ and $v_{r}$ be the two highest (with respect to the partial ordering) vertices in $\partial D$ other than the unique maximum vertex $v$. They are on opposite sides of $\partial D$, and are thus incomparable in $\partial D$, but not necessarily in $K_{i}$. If $v_{l}$ and $v_{r}$ are the same vertex in $K_{i}$, we join them by an arc in 
$D$ and then collapse this arc to produce $K_{i+1}$. Otherwise, assume they are different in $K_{i}$.

It is possible that a directed path $\alpha_{l}$ exists from $v_{r}$ to $v_{l}$ in $K_{i}^{1}$, or a path $\alpha_{r}$ from $v_{l}$ to $v_{r}$, but not both, since $K_{i}^{1}$ contains no directed loops, and the vertices are distinct. Suppose without loss of generality that $\alpha_{l}$ does not exist.

Then if we choose a point $p$ in the midpoint of the directed arc from $v_{l}$ to $v$, join $p$ to $v_{r}$ by an arc in $D$, and collapse this arc to produce $K_{i+1}$, the resulting oriented 1-skeleton will still be partially ordered. For, if such a directed loop $\alpha$ exists, then there is a directed path in $K_{i}^{1}$ from $v_{r}$ to $p$ or from $p$ to $v_{r}$. A directed outgoing arc from $p$ must pass through $v$, so in the second case we would have a directed path from $v$ to $v_{r}$. But there is a directed path from $v_{r}$ to $v$, which gives a directed loop in $K_{i}^{1}$, a contradiction. Similarly, an incoming loop to $p$ must pass through $v_{l}$, so in the first case we would have a directed path from $v_{r}$ to $v_{l}$. But by our assumption, no such $\alpha_{l}$ exists. Hence $K_{i+1}^{1}$ is partially ordered, and the induction step is complete. Of course, if no such $\alpha_{r}$ existed, we could have chosen $p$ as the midpoint of the arc from $v_{r}$ to $v$.

We see that there is a great deal of geometric control in this process: we get to choose the 2-cell we want to subdivide, and subdivide it by a definite amount, dividing it into a small bigon and another cell with one fewer vertices (of course, bigons can be completely collapsed!). Therefore it only takes a finite number of steps to collapse any compact region to a stage where the edges are all of length $<$ any $\epsilon$.

We take an exhaustion of $\tilde{M}$ by compact sets $C_{1} \subset C_{2} \subset \ldots$. Then we shorten every edge of $C_{1}$ to length $2^{-1}$, then every edge of $C_{2}$ to length $2^{-2}$, and so on until we eventually shorten any given edge to less than $\epsilon$ in finite time. By uniformity of this collapse we can pass to a limit. In this limit every 2-cell has been collapsed, and the limiting object is an infinite 1-manifold. It is clear from the construction that each edge is embedded by an orientationpreserving embedding. The associated embeddings of the upper and lower edge of every triangle in the long edge of the triangle are compatible across every tetrahedron, and the induced foliation of $\tilde{M}^{2}$ therefore extends to a transverse foliation of $\tilde{M}$. The local condition at vertices implies that this foliation is non-singular.

By construction, this foliation has no holonomy, so it admits a transverse measure.

Remark 5.1. Suppose that $M$ is hyperbolic, and suppose that it is trian- 
gulated by geodesic simplices. There is an $\epsilon$ and a $c$ such that if one can show that every edge has length $\geq c$ and the angle defect at an oriented angle (i.e. between an incoming and an outgoing edge to a vertex) is $\leq \epsilon$, then every oriented loop is homotopically essential. To see this, observe that a piecewise geodesic in $\mathbb{H}^{2}$ whose edges are all length $c$ and whose angle defects are all $\epsilon$ is an embedded quasigeodesic when

$$
\frac{\pi-\epsilon}{2}>\sin ^{-1}\left(\frac{1}{\cosh c / 2}\right)
$$

Any choice of $\epsilon, c$ satisfying the inequality above will work, by a comparison argument.

More generally, given geometric control on $M$, it is possible in certain circumstances to verify that every oriented loop is homotopically essential by showing that every oriented subarc of length $<K$ for some sufficiently large constant is quasi-geodesic with a sufficiently small coefficient of quasigeodicity. Since such conditions are merely sufficient but not necessary, we do not pursue this point.

The previous theorem did not require the local orientation on $M$ to be recurrent. However, that condition is critical for the next theorem.

Theorem 5.2. Let $M$ admit a recurrent local orientation in which every oriented loop is homotopically essential. Suppose $\tilde{M}$ is transversely measured by $f: \tilde{M} \rightarrow \mathbb{R}$ normal on every tetrahedron, and non-singular everywhere. Suppose $i: D \rightarrow \tilde{M}$ has boundary contained in a leaf. Then $i$ can be homotoped rel. $\partial D$ to map $D$ entirely into that leaf.

Remark 5.2. This somewhat technical theorem is essential to what follows, and constitutes the analogue in our context to the "main step" of the proof of Novikov's theorem; see [No].

Remark 5.3. Since a normal foliation is determined up to normal isotopy by its intersection with the 1-skeleton, we can straighten this foliation on each simplex in such a way that each normal disk is a flat triangle or a quadrilateral made up of 4 flat triangles, with respect to some equivariant affine structure on each tetrahedron pulled back from $M$. We assume below that this has been done.

Remark 5.4. A posteriori, using Rosenberg's theorem, it will be shown that the disk $D$ can be isotoped into a leaf, and not merely homotoped. 
Proof. Since the homotopy property described above is open, it suffices to show that a limit of disks which can be homotoped can itself be homotoped.

First observe that since each leaf is a normal surface, any sufficiently small disk with boundary on a leaf can be isotoped into that leaf.

Assume that $\left.f\right|_{D}$ has the following form.

- $f \circ i(\partial D)=1$ and $f \circ i(D)<1$ on the interior

- $f$ has exactly one critical point, a minimum, on $i(D)$

- the preimages $f^{-1}(p) \cap i(D)$ foliate $i(D)$ by concentric circles, nesting about this minimum

- the minimum of $f$ on $i(D)$ is 0

If we can show that the theorem holds for $D$ with $\left.f\right|_{D}$ of this form, then we can show that it holds inductively for all $D$, by successively pushing in innermost disks foliated as above, and reducing the number of critical points of $\left.f\right|_{D}$. Such a sequence of moves might involve self-intersections of $i(D)$ with itself (i.e. it might be a homotopy rather than an isotopy) but it will be a homotopy through immersions, since it restricts either to an isotopy or to the identity on each piece, at each time.

We denote

$$
C_{t}=i(D) \cap f^{-1}(t), D_{t}=i(D) \cap f^{-1}([0, t])
$$

and we let $E_{t}$ be the disk, a subset of $f^{-1}(t)$, whose boundary is $C_{t}$, for $t<1$. The existence of $E_{t}$ is guaranteed by hypothesis.

If the $E_{t}$ lie in a compact subset of $\tilde{M}$, then their limit $E$ exists and is a disk, since each $E_{t}$ is a normal surface. Since by hypothesis, no such. disk $E$ exists, we must suppose that the $E_{t}$ go off to infinity and the leaf $E$, the subset of $f^{-1}(1)$ bounded by $i(\partial D)$, is noncompact. (In case $i(\partial D)$ is non-separating, we can consider instead $E_{t}$ for $t$ extremely large, so that $E_{t}$ can be assumed to leave any compact neighborhood of $i(D)$ that we choose.)

We establish the following lemma

Lemma 5.3. In the above context there is a $K$ such that for every normal subdisk $D$ of a leaf whose boundary bounds another normal disk $D^{\prime}$ in $M$, such that $D$ and $D^{\prime}$ are homologous, if $|D|$ denotes the number of normal disks in $D$, we have an inequality

$$
|D| \leq K\left|D^{\prime}\right|
$$


Proof. Let $\alpha$ be a loop in the 1-skeleton of $M$ which passes through every edge. We can find such an $\alpha$ by the assumption that the 1-skeleton of $M$ is recurrent. Since $D, D^{\prime}$ were assumed to be homologous, the surface $D \cup D^{\prime}$ is null-homologous in $\tilde{M}$ and it therefore projects to a null-homologous surface in $M$, whose algebraic intersection number with $\alpha$ is therefore zero. Now, there is some constant $c_{1}>0$ such that the geometric intersection $\left|\alpha \cap D^{\prime}\right| \leq c_{1}\left|D^{\prime}\right|$, since $\alpha$ passes only finitely many times through each edge in $M^{1}$. Moreover, since $D$ is a subdisk of a leaf, the geometric intersection of $\alpha$ with $D$ is equal to their algebraic intersection, which is at least $c_{2}|D|$ for some $c_{2}>0$ depending on the maximal order of an edge in $M^{1}$. From $c_{1}$ and $c_{2}$ we can find our constant $K$.

Now, each leaf $E_{t}$ in homologous to some subdisk of $i(D)$. By simplicial approximation, these subdisks of $i(D)$ are all approximated by normal disks of bounded size. By our lemma, therefore, there is a bound on the number of normal disks in $E_{t}$, contradicting our assumption that the $E_{t}$ went off to infinity. This contradiction establishes the theorem.

Corollary 5.4. Every leaf in $\tilde{M}$ as above is incompressible.

Proof. This is immediate by the loop theorem.

Corollary 5.5. $M$ as above is irreducible or $S^{2} \times S^{1}$.

Proof. If $M \neq S^{2} \times S^{1}$ and it is reducible, then there is an embedded separating sphere in $M$. This lifts to an embedded sphere in $\tilde{M}$. Then our result follows word for word the proof of Rosenberg's Theorem [Ro], after remarking that every leaf in $\tilde{M}$ is a possibly disjoint union of $\mathbb{R}^{2}$ 's and $S^{2}$ 's. If any leaf contains an $S^{2}$, the Reeb stability theorem, together with our result, shows that $\tilde{M}$ is $S^{2} \times \mathbb{R}$ (since, inductively, no "first" leaf can become non-compact). Since $M$ is compact and orientable, it is $S^{2} \times S^{1}$ or $\mathbb{R} P^{3} \# \mathbb{R} P^{3}$. But if $M=\mathbb{R} P^{3} \# \mathbb{R} P^{3}$ then as in the previous section the image of an $S^{2}$ separates $M$ and contradicts recurrence. If $\tilde{M}$ is foliated by $\mathbb{R}^{2}$ 's it is irreducible.

Corollary 5.6. If $\tilde{M}$ is $\delta$-hyperbolic with respect to (any) complete metric pulled back from $M$, then each leaf in $\tilde{M}$ foliated as above by incompressible leaves is $\delta^{\prime}$-hyperbolic, where $\delta^{\prime}$ depends only on $\delta$ and the combinatorics of 
$M$.

Proof. From the proof and the statement of theorem 5.2 we can deduce that for every normal subdisk $D$ of a leaf whose boundary bounds another normal disk $D^{\prime}$ in $\tilde{M}$, we have an inequality $|D| \leq K\left|D^{\prime}\right|$ for some $K$ depending only on the triangulation of $M$. For, $\pi_{2}(\tilde{M})=H_{2}(\tilde{M})=1$, and therefore any two disks in $\tilde{M}$ with the same boundary are homologous.

Now, we know that $\delta$-hyperbolic Riemannian manifolds are characterized by the fact that minimal spanning disks satisfy linear isoperimetric inequalities (see, for instance, $[\mathrm{Gr}]$ ). Our comparison lemma allows us to establish a similar isoperimetric inequality for subdisks of a leaf, and therefore each leaf is also $\delta^{\prime}$-hyperbolic. One can see that $\delta^{\prime}$ depends only on the combinatorics of $M$ and on $\delta$.

Remark 5.5. Note that Rosenberg's theorem shows that disks can be isotoped into leaves, rel. boundary. For if $D_{1} \simeq D_{2}$ rel. boundary, we can consider intersections $D_{1} \cap D_{2}$, which will be a collection of circles. But innermost circles in the intersection will define $S^{2}$ 's in $D_{1} \cup D_{2}$ which must bound $B^{3}$ 's. Pushing $D_{1}$ across this $B^{3}$ we can isotope it to reduce the number of components of $D_{1} \cap D_{2}$. Inductively, this shows we can isotope $D_{1}$ to $D_{2}$ rel. boundary.

Remark 5.6. Notice that the hypothesis that every oriented loop in $M$ be homotopically essential is used only to establish the existence of a foliation on $\tilde{M}$ in normal form, transverse to the orientations pulled back from $M$. If we are given this foliation on $\tilde{M}$ as a hypothesis, say if the orientation on $M$ was inherited from a foliation in the first place, then the proof of the theorem still goes through, and we can show that every oriented loop in $M$ is homotopically essential as a consequence of the existence of the foliation. on $\tilde{M}$. Thus, our technique gives a new proof (conceptually similar, though. perhaps technically easier) of the theorem of Novikov that circles transverse to taut foliations are homotopically essential.

More explicitly, given a taut foliation on $M$, we can take a sufficiently fine triangulation and orientations on the edges to obtain a local orientation. Lifting this foliation to the universal cover, our theorem applies to show that every leaf in $\tilde{M}$ is incompressible and is therefore a disk or a sphere. Such a foliation has no holonomy, so it admits a transverse measure. Since we are on $\tilde{M}$, a transverse measure is given by integrating an exact 1 -form $d f$. A homotopically inessential transverse circle in $M$ lifts to a transverse circle in 
$\tilde{M}$. But $d f$ is positive everywhere on the tangent vector to this circle which is absurd.

Remark 5.7. Our result shows that the foliations on the universal cover are, roughly speaking, minimal surfaces with respect to the weights on edges determined by an oriented cycle in $M$ passing through every edge. This is in some sense a combinatorial (non-deterministic) volume-preserving flow on $M$. This flow is used by Gabai in [Ga2] to prove a refinement of a theorem of Roussarie and Thurston: if $S$ is an immersed incompressible surface in $M$ admitting a taut foliation, then $S$ can either be homotoped into a leaf, or can be homotoped to have only saddle-type tangencies with the foliation.

We can produce a qualitative topological refinement of theorem 5.2 by a more delicate argument.

Theorem 5.7. Suppose $M$ is not prime, and suppose its 1-skeleton is ordered in such a way that all the conditions of the above theorem except recurrence are satisfied. Then there exists an embedded null-homologous cooriented (not necessarily connected) normal surface in $M$ such that every transverse arc is outgoing.

Proof. As in theorem 5.1 we can produce a measured foliation on $\tilde{M}$. If every leaf were incompressible, $M$ would be prime. Therefore some leaf is compressible, and by the loop theorem, there is an embedded disk $D$ in $\tilde{M}$ whose boundary lies on a leaf, such that there is no disk in that leaf with the same boundary. We adopt the notation from the proof of theorem 5.2. and assume the restriction of the foliation to $D$ is by concentric circles, by induction.

Recall that $D$ is a disk whose boundary lies in a leaf of the foliation of $\tilde{M}$, that $E$ is the subset of this leaf bounded by $\partial D$, and that $E$ is a "limit" of the $E_{t}$ - disks contained in the foliation whose boundaries are circles making up a concentric foliation of $D$.

If $R$ is the region in $\tilde{M}$ bounded by $D$ and $E$ then every increasing arc that passes through $E$ must leave $R$. Now, $E$ is made up of normal triangles and quadrilaterals, so there are outgoing arcs within bounded distance of any point on $E$. Since the only incoming arcs to $R$ pass through $D$, and since every vertex is the endpoint of some arc, there are arbitrarily long paths contained in $\tilde{M}^{1}$ passing through $D$ and contained entirely within $R$. Since each sufficiently long arc eventually passes through each $E_{t}$, and since each $E_{t}$ bounds a compact region of $R$, we can extract a subsequence of 
these paths which converge on compact sets (since they are all simplicial!), and find an infinite increasing path $\gamma \in \tilde{M}^{1}$ whose initial point is in $D$ and which is contained entirely inside $R$. Notice that $\gamma$ intersects each $E_{t}$ exactly once for all $t>t_{0}$, the value of $f$ at the initial point of $\gamma$. Let $\gamma_{t}$ denote the initial segment of $\gamma$ from 0 to $t$.

Since the $D_{t}$ converge to $D$, there is some $t$ after which all the $C_{t}$ are normally isotopic to $\partial D$. If we truncate $R$ by only considering the region above this $t$, we can replace $D$ by a slight perturbation of $E_{t}$, and therefore we can assume, without loss of generality, that every increasing simplicial arc passing through $D$ is incoming to $R$.

By the compactness of $M$, we can assume there is some $p \in M^{0}$ such that $\gamma$ passes through infinitely many lifts of $p$ which we call $p_{0}, p_{1}, \ldots$ Let $\alpha_{i} \in \pi_{1}(M)$ be such that $\alpha_{i}\left(p_{i}\right)=p_{0}$. After passing to a subsequence and re-ordering if necessary, we can assume that the translates $\alpha_{i}(D)$ are disjoint and the collection is embedded.

Since our foliation of $\tilde{M}$ is not necessarily $\pi_{1}(M)$ equivariant, the image of subsets of the leaves can intersect. We need to investigate these intersections more closely. We have the following lemma, which controls the orientations on $\alpha_{i}\left(E_{t}\right), \alpha_{j}\left(E_{s}\right)$ when they intersect in an isolated point of tangency.

Lemma 5.8. If some normal subsurfaces of $\alpha_{i}\left(E_{t}\right), \alpha_{j}\left(E_{s}\right)$ intersect in an isolated point of tangency for some $i, j, s, t$ then the transverse orientations to $\alpha_{i}\left(E_{t}\right), \alpha_{j}\left(E_{s}\right)$ agree at this point.

Proof. If the intersection is at a vertex, then a neighborhood of the intersection in either surface separates the star of the vertex. By the definition of the foliation on $\tilde{M}$, the outgoing and the incoming edges to the vertex lie in different components of the star. Since both these collections are nonempty, there is a monotone arc transverse to both surfaces at the point of intersection, whose orientation agrees with the transverse orientations on both surfaces at this point.

If the intersection is in some tetrahedron $\Delta$, then there are a pair of normal disks in $\alpha_{i}\left(E_{t}\right) \cap \Delta, \alpha_{j}\left(E_{s}\right) \cap \Delta$ which intersect in an isolated point of tangency. Since any two normal disks in a tetrahedron intersect a common edge, this edge is transverse to both surfaces at this point and their transverse orientations therefore agree.

Since each $\alpha_{i}\left(\gamma_{t}\right)$ must pass through $p_{0}$ for some $t$, and since $\alpha_{i}\left(\gamma_{t}\right)$ 
lies outside $R$ for sufficiently small $t$, there is some increasing sequence $t_{i}$ such that $\alpha_{i}\left(\gamma_{t_{i}}\right)$ intersects $D$. As we increase $t$ past $t_{i}$, the surface $\alpha_{i}\left(E_{t}\right)$ intersects a collar neighborhood of $D$ in an annulus insulating $\alpha_{i}(\gamma)$ from $\partial D$, and expanding concentrically with $t$. Let $B_{i, 0}(t)$ be the circle of intersection with $D_{t}$ for $t$ near $t_{i}$. Then as $t$ increases, we may push part of $B_{i, 0}(t)$ over the edge of $D_{t}$ and up into $E_{t}$. That is, we think of $B_{i, 0}(t)$ as the appropriate component of $\alpha_{i}\left(E_{t}\right) \cap\left(E_{t} \cup D_{t}\right)$.

Lemma 5.9. There is some finite $n$ such that there are at most $n$ embedded circles and properly embedded arcs made from pieces of $\alpha_{i}\left(E_{t}\right) \cap D$ extending normally to a simplicial collar of $D$ such that no two of the collection are normally isotopic in a neighborhood of $D$.

Proof. These circles and arc are the meridians of embedded normal annuli and arcs $\times I$ transverse to $D$. Therefore they bound embedded subdisks of $D$, and since they are normal, the length of the circles and arcs is bounded. Hence each annulus and arc $\times I$ is composed of a bounded number of pieces, and since the simplicial neighborhood is finite, there are only finitely many normal surface types represented by them.

Notice that since $t_{j}<t_{i}$ for $j<i$, the surface made up from $\alpha_{j}\left(E_{t}\right) \cup$ $\alpha_{j}(D)$ for appropriate $t$ separates $\alpha_{i}(D)$ from $p_{0}$ for $i>j$. In particular, $\alpha_{i}\left(\gamma_{t}\right)$ must pass through each $\alpha_{j}(D)$ for $j<i$ before passing through $D$. Suppose $\alpha_{i}\left(\gamma_{t}\right)$ exits $\alpha_{j}\left(E_{t}\right)$ for some $j<i$ and $t<t_{i}$. Then again it can never reach $p_{0}$. We denote the circles of intersection of the $\alpha_{i}\left(E_{t}\right)$ with $\alpha_{j}\left(E_{t} \cup D_{t}\right)$ by $B_{i, j}(t)$. Notice that

$$
B_{i, i}(t)=\alpha_{i}\left(\partial D_{t}\right)
$$

which is normally isotopic to $\alpha_{i}(\partial D)$ for large $t$.

The $B_{i, j}(t)$, with $i \leq k$ divide $\alpha_{j}(D)$ for fixed $j$ into a collection of regions. By our previous comment, each $B_{i, j}(t)$ bounds the region containing $\alpha_{k}(\gamma) \cap \alpha_{j}(D)$ for $i \leq k$. Let $B_{j}^{k}(t)$ be the boundary of the subregion of $D$ containing $\alpha_{k}(\gamma) \cap \alpha_{j}(D)$. This is a circle contained entirely within $\alpha_{j}(D)$. Call $B_{j}^{k}(t)$ an innermost circle.

Since there are only finitely many possibilities for the $B_{j}^{k}(t)$ for each $j$, up to normal isotopy, by choosing $i$ very large we can find $B_{j}^{i}(t)$ such that $\alpha_{k} \circ \alpha_{j}^{-1}\left(B_{j}^{i}(t)\right)$ is normally isotopic to $B_{k}^{i}(t)$. We know that the innermost circle $B^{\prime}$ made up of $B_{l, k}(t)$ with $j \leq l \leq i$ lies outside $B_{k}^{i}(t)$, since it is 
the innermost of fewer circles. By definition, there is an annulus made up of pieces of $\alpha_{i}\left(E_{t}\right)$ interpolating between $B_{j}^{i}(t)$ and $B^{\prime}$. This annulus is embedded, since it bounds some image of $\gamma$, and we can take an innermost such. In more detail, this annulus is the boundary of the connected region in the complement of the relevant $\alpha_{i}\left(E_{t}\right)$ which bounds the relevant image of $\gamma$. Since each $\alpha_{i}\left(E_{t}\right)$ bounds this image of $\gamma$, such a region exists. Our orientation lemma implies it is an annulus.

Since each $B_{i, k}(t)$ bounds some subdisk of $\alpha_{i}\left(E_{t}\right)$, we can cut and paste an innermost disk which bounds $B^{\prime}$. Together with the subdisk $D^{\prime}$ of $\alpha_{j}\left(D_{t}\right)$ bounded by $B_{j}^{i}(t)$ this gives a (topological) sphere $S$ bounding a $B^{3}$ in $\tilde{M}$ such that $B_{k}^{i}(t)$ and the subdisk $\alpha_{k} \circ \alpha_{j}^{-1}\left(D^{\prime}\right)$ of $\alpha_{k}\left(D_{t}\right)$ that it bounds is entirely contained inside the region bounded by $S$. One should be careful to note that the sphere in question bounds a $B^{3}$ because it is contained in the region $R$ whose interior is foliated with disks, and therefore irreducible.

Let $\beta=\alpha_{k} \circ \alpha_{j}^{-1}$, and let $N=\tilde{M} /<\beta>$. Then since $S$ bounds a ball in $\tilde{M}$, its image under the projection to $N$ is some compact submanifold on $N$. Its boundary cannot contain any piece of $D^{\prime}$, since $D^{\prime}$ is interior to some translate of the ball bounded by $S$. If $N$ is non-compact, this boundary is non-empty and by construction is a null-homologous normal surface in $N$ made up entirely of projections of pieces of $E_{t}$. Call this surface $H$ and consider its projection $\pi(H)$ to $M$.

If the boundary is empty, then $N$ is compact, and $M$ is $S^{2} \times S^{1}$ by an earlier result.

Since $\pi(H) \subset M$ is made up entirely of pieces of the image of $E_{t}$ under the projection $\tilde{M} \rightarrow M$, it is represented by an embedded (possibly disconnected) normal surface $G=G_{1} \cup G_{2} \cup \cdots \cup G_{n}$. By our orientation lemma, each $G_{i}$ is co-oriented such that every transverse arc in the 1-skeleton is outgoing.

Remark 5.8. This theorem slightly weakens the condition of recurrence to prove irreducibility.

Notice that such a surface $G$ is a finite Haken sum of fundamental normal surfaces co-oriented compatibly with the orientation on the 1-skeleton. One can check algorithmically whether some $\mathbb{Z}$-linear combination of such fundamental surfaces can be trivial in $\mathrm{H}_{2}$. 


\section{Is Homotopically Essential Essential?}

In this section we show that, at least to prove irreducibility, the condition that every oriented loop in the 1-skeleton be homotopically essential can be substantially weakened, and even weakened to an easily checkable condition.

Definition 1. Let $M$ be a triangulated 3-manifold. For any $m \in \mathbb{Z}$, the simple combinatorial $m$-germ at a vertex $p$, denoted $\tilde{M}_{m}(p)$, is the simplicial complex obtained in the following way:

- Let $N_{m}(p)$ be the disjoint union of the simplicial neighborhoods of simplicial paths of length $m$ in $M$ with initial vertex $p$.

- Obtain $\tilde{M}_{m}(p)$ as the quotient space of $N_{m}(p)$ by identifying endpoints of two distinct paths which have the same endpoint in $M$, and which bound an (immersed) simplicial disk in $M$ of simplicial area $\leq m$.

Remark 6.1. It is clear from the definition that the complex $\tilde{M}_{m}(p)$ can be algorithmically constructed. Note that we could fine-tune the relative sizes of paths and disks in the definition to more accurately capture approximations to the germ of $\tilde{M}$ at $p$ using estimates of an isoperimetric inequality for $M$.

Theorem 6.1. Let $M$ admit a local orientation such that for an appropriate, explicitly computable constant $k$ depending only on the triangulation and the orientation of $M$, the complex $\tilde{M}_{k}(p)$ with the induced orientations on the 1-skeleton has no oriented loops. Then $\pi_{2}(M)=1$ or $M=S^{2} \times S^{1}$.

Proof. If $M$ is not $S^{2} \times S^{1}$ and is reducible, then there exists a separating normal $S^{2}$. This $S^{2}$ lifts to $\tilde{M}_{k}(p)$ for sufficiently large $k$. If $\tilde{M}_{k}(p)$ has no oriented loops, it admits a transverse co-oriented foliation in normal form.

As in the proof of our earlier theorem, we consider the intersection of the $S^{2}$ with this foliation, and start to push innermost disks into the leaves. Our earlier estimates for the simplicial size of these disks still holds, since $M^{1}$ is recurrent. Every subdisk of the homotopy is within an (easily computable) distance from some fixed $p \in S^{2}$, and all the disks that we are pushing are of simplicial size bounded by some computable constant times the simplicial size of the $S^{2}$, so this homotopy can be carried out within $\tilde{M}_{k}(p)$ (i.e. we never push over the boundary). 
If this $S^{2}$ can be pushed entirely into an $S^{2}$ leaf, then there is a separating $S^{2}$ in $M$ oriented compatibly with the 1-skeleton, which contradicts recurrence. Otherwise, the $S^{2}$ can be pushed entirely into a disk, and therefore was null-homotopic in $M$.

The theorem is proved once we observe that we can bound the simplicial size of the smallest separating embedded normal homotopically essential $S^{2}$ in terms of the triangulation of $M$.

\section{Questions.}

It is natural to ask to what extent some of the technical hypotheses in this paper can be removed. In particular, the following questions seem outstanding:

1. Is there an algorithm to check whether every oriented loop in a local orientation is homotopically essential?

2. To what extent is our combinatorial structure weaker than the existence of a taut foliation in normal form?

Remark 7.1. The referee has pointed out that good candidates for local orientations with homotopically essential loops which do not admit transverse foliations might be found by investigating certain graph manifolds, in particular those obtained from products (punctured surface) $\times S^{1}$ by appropriate glueings along the boundary tori. Work of Brittenham, Naimi and Roberts [BNR] shows that many such graph manifolds do not admit any taut foliations whatsoever. On the other hand, such manifolds certainly admit local orientations, and one expects that the condition that oriented loops be homotopically essential can be satisfied in many cases.

3. If a triangulation of $M$ admits a local orientation in which every oriented loop is homotopically essential, to what extent can this combinatorial structure be extended over a refinement of the triangulation?

4. Is there some (computable) bound on the number of subdivisions of a triangulation necessary to put it in normal form with respect to an existing $\left(C^{1}\right)$ foliation on $M$ ?

5. Can the finiteness of the combinatorial structure be used to advantage in addressing questions of the virtual existence of such a structure - 
i.e., when does there exist a finite cover of $M$ which admits a local orientation in which each loop is homotopically essential? or lies in an open half-space of $H_{1}$ ?

Remark 7.2. This question is intimately related to Thurston's famous conjecture that every hyperbolic 3-manifold has a finite cover which fibers over the circle. In fact, this question was our main original motivation for studying the interaction of foliations with finite combinatorial structures.

6. If $\alpha$ is an oriented embedded loop in $M^{1}$ which has a local orientation in which each oriented loop is homotopically essential, is it true that all Dehn surgeries on $\alpha$ with slope sufficiently close to $(1,0)$ give manifolds whose 1-skeleton can be similarly oriented without changing the triangulation or the orientation on $M-\operatorname{nbhd}(\alpha)$ ?

7. To what extent can the geometry of leaves in $\tilde{M}$ be controlled?

8. Can one extend the results of the last few sections to triangulations with local orientations on subgraphs of the 1-skeleton?

Remark 7.3. Our local orientations are dual to branched surfaces such that complementary domains are sutured balls. More generally, branched surfaces whose complementary domains are sutured manifolds admitting taut foliations can (with certain technical hypotheses) carry only incompressible surfaces (see [Oe]). It seems plausible that if one controls the complementary regions, homotopically essential local orientations on subsets of $M^{1}$ might have many nice properties.

\section{References.}

[Be] M-T. Benameur, Triangulations and the Stability Theorem for Foliations, Pacific J. Math. 179 (1997), 221-239.

[BNR] M. Brittenham, R. Naimi and R. Roberts, Graph Manifolds and Taut Foliations, J. Diff. Geom. 45 (1997), 446-470.

[Ga] D. Gabai, Problems in Foliations and Laminations, in Geometric Topology, (edited by W. Kazez), proceedings of the 1993 Georgia International Topology Conference, Vol. 2, part 2, 1-33.

[Ga2] D. Gabai, A Combinatorial Volume Preserving Flow on Taut Foliations, preprint. 
[Gr] M. Gromov, Hyperbolic Groups, in Essays in Group Theory, MSRI Publ. 8 (1987), 75-263.

[No] S. Novikov, Topology of Foliations, Trans. Moscow Math. Soc. 14 (1965), 268-305.

[Oe] U. Oertel, Homology Branched Surfaces: Thurston's Norm on $\mathrm{H}_{2}\left(M^{3}\right)$, in Low-Dimensional Topology and Kleinian Groups, LMS Lecture Note Series 112 (1986), 253-272.

[Ro] H. Rosenberg, Foliations by Planes, Topology, 7 (1968), 131-138.

[Su] D. Sullivan, Cycles for the Dynamical Study of Foliated Manifolds and Complex Manifolds, Inventiones Math., 36 (1976), 225-255.

ReCEIVEd DeCember 5, 1997.

UC BERKELEY

BERKELEY, CA 94720

E-mail address: dannyc@math.berkeley.edu 